La Revue

des Droits

de l'Homme

\section{La Revue des droits de l'homme}

Revue du Centre de recherches et d'études sur les droits fondamentaux

13 | 2018

Revue des droits de l'homme $-\mathrm{N}^{\circ} 13$

\title{
Entretien avec Pascal Brice, Directeur général de l'OFPRA : « Entre continuité et modernisation : la diversification des missions de l'OFPRA »
}

\section{Marion Tissier Raffin}

\section{(2) OpenEdition \\ Journals}

Édition électronique

URL : http://journals.openedition.org/revdh/3681

DOI : $10.4000 /$ revdh.3681

ISSN : 2264-119X

Éditeur

Centre de recherches et d'études sur les droits fondamentaux

Référence électronique

Marion Tissier Raffin, « Entretien avec Pascal Brice, Directeur général de l'OFPRA : « Entre continuité et modernisation : la diversification des missions de l'OFPRA » », La Revue des droits de l'homme [En ligne], 13 | 2018, mis en ligne le 05 janvier 2018, consulté le 08 juillet 2020. URL : http:// journals.openedition.org/revdh/3681 ; DOI : https://doi.org/10.4000/revdh.3681

Ce document a été généré automatiquement le 8 juillet 2020.

Tous droits réservés 


\title{
Entretien avec Pascal Brice, Directeur général de l'OFPRA : «Entre continuité et modernisation : la diversification des missions de l'OFPRA »
}

\author{
Marion Tissier Raffin
}

1 Pour commencer ce numéro spécial de la Revue des Droits de l'Homme, nous avons voulu revenir sur les missions de l'office français de protection des réfugiés et apatrides (OFPRA) dans le contexte de la crise des réfugiés, et notamment sur l'intensification et la diversification de ses missions. À cette fin, quoi de plus pertinent que de s'entretenir avec son Directeur général, Pascal Brice, qui occupe cette fonction depuis 2013. Nous en avons également profité pour recueillir son analyse de la crise des réfugiés en France et son point de vue sur le développement croissant de procédures ad hoc de protection des réfugiés.

2 Thème 1 - L'intensification et la diversification des missions de l'OFPRA : " la mission de l'OFPRA, c'est de faire vivre le droit d'asile ", Pascal Brice.

Depuis 2013, les missions de l'OFPRA sur le sol national se sont diversifiées? Pouvez-vous revenir sur ces missions et sur les raisons qui ont conduites l'OFPRA à sortir de ces murs?

Depuis deux à trois ans, nous avons voulu que l'OFPRA fasse preuve d'une plus grande réactivité.

5 Le constat que nous avons fait est le suivant. Tout d'abord, d'un point de vue migratoire, nous avons constaté qu'il y avait un nombre croissant de demandeurs d'asile - même si je maintiens que ce nombre ne signifie pas qu'il y ait un afflux massif un déficit d'information de la part des demandeurs d'asile - même si la procédure d'asile a été simplifiée - , et des demandeurs d'asile de plus en plus mobiles. À cela s'ajoute le contexte social général du pays qui suppose une forte mobilisation des acteurs publics dans le pays, de manière à faire la démonstration que le droit d'asile est 
pleinement respecté et qu'il l'est dans l'efficacité. L'ensemble de ces raisons nous a conduit a finalement revenir à ce qui était l'histoire de l'OFPRA il y a une vingtaine d'années, en multipliant les missions dans le pays.

Plus spécifiquement, l'acte fondateur a été l'intervention de l'OFPRA à l'été 2013 premier moment de la crise de l'asile - à St Ouen, lorsque plusieurs familles syriennes en très grande précarité s'y sont installées. Nous y sommes allés pour les rencontrer, convaincus qu'elles pouvaient relever de l'asile - sous réserve de l'instruction. Et c'est ainsi que nous avons mis en place un dispositif spécifique très intégré avec l'office français de l'immigration et de l'intégration (OFII) et la préfecture. C'était la première opération de ce genre. Depuis, ce type de mission n'a cessé de se développer.

作 d'instruction. En effet, il faut rappeler que l'OFPRA n'est pas saisie en début de procédure d'asile; elle ne l'est qu'en second lieu, après le passage en préfecture. Ainsi, en 2016, nous avons effectué quarante-deux missions, sans compter les missions hebdomadaires à Calais et à Grande Synthe. Pourtant, la mission fondamentale de l'OFPRA reste l'instruction de la demande d'asile. Il ne faut donc pas confondre les missions d'information et les missions d'instruction.

8 Pour les missions d'information, l'exemple le plus emblématique reste celles menées à Calais entre le printemps 2014 et la fin 2016. L'OFPRA était présente deux fois par mois, voire toutes les semaines. L'information portait essentiellement sur l'accès à la demande d'asile. Et je dois dire que le résultat est satisfaisant. Avant 2014, et même avant 2015, il n'y avait quasiment pas de demandeurs d'asile à Calais. Notre grande fierté est d'avoir installé, avec les associations, une véritable culture de l'asile. Près de 10000 personnes ont obtenu l'asile. L'intuition initiale à Calais était donc la bonne. Il y a aussi eu des missions d'information à Grande Synthe et dans le centre de Paris, Porte de la Chapelle. C'est ce même type de missions d'information que nous réalisons dans les hot spots en Italie (nous y revenons un peu plus loin).

9 Toutefois, comme je l'ai dit, le cœur de notre activité, ce sont les missions d'instruction. Les modalités d'organisation de ces missions d'instruction se sont elles aussi diversifiées puisqu'elles se déroulent de plus en plus en dehors du siège de Fontenay. Ainsi, nous les réalisons, soit dans les régions, y compris en outre-mer (Guyane et Mayotte) et à Athènes (relocalisation: une mission mensuelle) mais aussi au ProcheOrient (Liban : Beyrouth ; Turquie : Ankara). Nous mettons en place ce type de missions lorsque nous constatons (propres informations ou remontées d'information par les associations/élus/préfet) qu'il y a un nombre important de demandeurs d'asile, souvent de même nationalité, regroupés sur un même espace géographique. Par exemple, à Bordeaux, il y avait un nombre important de Saharaouis; à Metz, c'étaient des d'Albanais et à Cayenne, des Haïtiens. À travers ces informations, nous comprenons qu'il y a des difficultés en termes d'accueil, d'accompagnement et d'hébergement. L'idée d'intervenir sur place est précieuse car elle évite d'abord aux demandeurs d'asile de se déplacer jusqu'à Paris. Elle nous met aussi en contact direct avec tous les acteurs locaux de l'asile (préfecture - élus - associations). Les missions durent en général quinze jours et les officiers de Protection sont présents sur place pour mener les entretiens. Ces missions d'instruction sont devenues un mode de fonctionnement régulier de l'office et elles sont un élément important de la réactivité et de l'efficacité de l'OFPRA. 
10 Finalement, le développement de ces missions d'instruction dites foraines est une réponse à une hypothèse récurrente et redondante dans l'histoire de l'OFPRA, celle de la mise en place d'antennes permanentes de l'office en région. La question est réapparue lors de la réforme de l'asile en 2015. Moi-même et l'OFPRA étions prêts à ouvrir des antennes en région, sous certaines conditions évidemment. La principale était que ce soit des antennes structurées de l'OFPRA, dotées de la même indépendance institutionnelle de l'OFPRA, notamment vis-à-vis des préfectures. Cela ne peut pas être deux ou trois agents isolés au milieu dans une préfecture. L'hypothèse de créer de telles antennes reste d'ailleurs ouverte. Mais, je pense que les missions foraines sont plus efficaces que les missions permanentes car elles permettent à l'OFPRA de s'adapter aux évolutions de la demande, c'est-à-dire de se déplacer là où les besoins se font sentir. Sachant que, sauf quelques rares exceptions comme à Lyon, il y a de réelles évolutions de la demande d'asile, en termes de volume, mais aussi en termes de localisations et de nationalités. Par conséquent, se positionner de manière permanente dans un lieu peut ne pas être un choix de bon sens sur le long terme. Les missions foraines répondent donc bien à cette stratégie d'efficacité et de réactivité évoquée précédemment.

11 Comment sont organisées les missions d'instruction des demandes d'asile déposées par des demandeurs d'asile résidant dans les Centres d'accueil d'orientation (CAO) ? Comment concilier l'objectif de l'OFPRA de faire vivre le droit d'asile, c'est-à-dire de procéder à l'instruction des demandes d'asile, et le non-respect plein et entier de la promesse de l'ancien gouvernement de ne pas appliquer la procédure Dublin pour ces demandeurs d'asile placés en CAO?

12 Tout d'abord, l'engagement de l'OFPRA était de procéder à des instructions rapides. Nous procédons alors de deux manières : soit des missions foraines sont organisées sur place (Lille - Nantes - Rennes - Bordeaux - Clermont-Ferrand - Mont-de-Marsan), soit les demandeurs d'asile sont conviés à un entretien au siège de l'OFPRA à Fontenay. (Exemples de jeunes afghans dans l'Allier).

13 En ce qui concerne la mise en œuvre de la procédure Dublin, il faut être clair. L'engagement précis de l'OFPRA à Calais, et du gouvernement, est le suivant : lorsque des personnes relèvent de la procédure Dublin et que les préfectures décident d'appliquer cette procédure, alors il ne peut pas y avoir de réadmission. Cela ne signifie pas «ne pas engager la procédure Dublin». Le seul engagement pris était "Pas de réadmission » et non " Pas de procédure Dublin ». Mais, il est bien évident que dans ce contexte-là, je ne vois pas l'intérêt d'engager la procédure Dublin. Je regrette l'ampleur des placements en procédure Dublin car je pense que cela n'a pas de sens et que cela complique la vie de tout le monde. Il faut donc veiller et être attentif à ce que les réadmissions n'aient pas lieu. Mais, comme je l'ai déjà dit, l'OFPRA ne peut instruire que lorsque la demande lui est transmise.

14 Quelle est votre analyse de l'avenir de Calais après le démantèlement de son bidonville, notamment après le refus de l'ancien gouvernement - désormais confirmé par le gouvernement actuel - de ne pas ouvrir de centre d'accueil pour les migrants? Et quel est le rôle de l'OFPRA sur le Calaisis?

15 Je souhaite que l'OFPRA n'ait pas à retourner à Calais. Je ressens une fierté d'avoir réussi collectivement une solution pour l'ensemble des personnes à Calais car, pour y avoir passé deux années, je sais de quoi je parle. Je n'ai aucune nostalgie de ce bidonville. J'ai même la satisfaction physique, en plein hiver, en plein froid, de me dire en sortant de chez moi que je ne les verrai pas aujourd'hui dans le bidonville. Les CAO sont les meilleurs visages de la France, même s'il a fallu parfois beaucoup de courage de 
la part des élus pour les mettre en place. Ils sont une formidable dynamique d'entraide et de solidarité de la part des français. C'est ce visage que je veux retenir.

Mais, nous savons tous que la question de Calais ne va pas disparaitre comme par enchantement. Il est donc nécessaire que des dispositifs soient mis en place pour celles et ceux qui pourraient continuer d'arriver à Calais, même s'il faut dire et redire que Calais est une impasse. Nous ne nous sommes pas battus pendant près de deux ans pour sortir de ce bidonville pour revenir sur ce discours. Il faut donc être très clair : aller à Calais ne sert à rien. Pour autant, pour celles et ceux qui continueraient d'y arriver, il faut un dispositif : si ce n'est pas un dispositif sur place, ce doit être un dispositif de prise en charge pour les faire entrer dans le dispositif national. Du point de vue de l'OFPRA, ce qui importe est de faire vivre l'asile là où il n'existe pas. Donc, il faut que les personnes qui arrivent à Calais puissent accéder, de quelle que manière que ce soit, à la demande d'asile. Pour les moyens, ils relèvent de la responsabilité du gouvernement.

Le plan de relocalisation adopté par l'Union européenne a conduit l'OFPRA à organiser ses missions en dehors du sol français, notamment en Grèce et en Italie. Quelles sont ces missions précises? Pourquoi ne sont-elles pas organisées de manière identique en Italie et en Grèce? Quels sont les critères d'identification et de sélection des personnes qui sont relocalisées?

Ce sont les Italiens ou les Grecs qui procèdent à l'identification des personnes en fonction d'un règlement européen pour les nationalités : syrienne et érythréenne (et avant, irakienne). Ce sont eux qui proposent les personnes éligibles à la relocalisation aux États d'accueil, et en l'occurrence à la France. Les personnes formulent quatre à cinq pays de destination. Il n'y a sur ce point pas de difficultés particulières (elles sont plus nombreuses en ce qui concerne les procédures de réinstallation). Et, dans le cadre de la relocalisation, les critères de vulnérabilité ne sont pas spécifiquement pris en compte comme cela peut l'être davantage pour la procédure de réinstallation. Il arrive que des associations nous alertent sur une vulnérabilité, mais, dans ce cas, cela se passe plutôt de manière informelle.

Le rôle de l'OFPRA dans le contexte de la relocalisation se situe plus précisément à deux niveaux :

Les officiers de protection de l'OFPRA, dans le cadre du bureau européen d'appui à l'asile, se trouvent en Italie - dans les ports exclusivement - et en Grèce - sur le continent exclusivement. L'OFPRA n'a en effet pas souhaité être présent dans les hot spots en Grèce. Nous sommes le premier contingent européen pour aider aux missions d'identification.

21 En Italie, une fois que les personnes sont proposées par les Italiens, les personnes viennent en France et c'est en France que se fait l'instruction. Le nombre de personnes proposées à la relocalisation depuis l'Italie et non éligibles au bénéfice d'une protection (internationale ou subsidiaire) après entretien/instruction en France, se compte sur les doigts d'une main. En Grèce, l'OFPRA se déplace quinze jours tous les mois à Athènes, ville où nous avons des locaux. Les entretiens sont alors faits sur place.

Ces différences s'expliquent par deux raisons principales :

- les Italiens n'ont pas souhaité faire ce que les Grecs ont accepté que nous fassions

- les enjeux sont différents en Grèce et en Italie, en termes de nationalités et de profils à risques d'exclusion. En Italie, la vigilance est renforcée.

De manière générale, les délais moyens entre le moment où il y a une proposition de relocalisation et l'effectivité du transfert sont de quatre à cinq mois. Ce sont donc des 
délais courts. Ces personnes sont alors directement placées en Centre d'accueil de demandeurs d'asile (CADA) : elles passent en guichet unique.

\section{affirmé que c'était l'une des conditions pour lever les réticences et les a priori à l'accueil des} demandeurs d'asile, voire un moyen de contourner le blocage des frontières. À votre avis, les procédures de relocalisation et/ou de réinstallation sont-elles amenées à se développer? Est-ce d'ailleurs souhaitable? Sont-elles l'avenir de la protection internationale des réfugiés?

Je pense que ces procédures ad hoc - relocalisation ou réinstallation- sont amenées à se développer et que cela est souhaitable, à une condition primordiale : elles ne doivent pas devenir la voie unique de protection des réfugiés. Je pense que ce sont des moyens de protection extrêmement précieux qu'il faut développer parce que, surtout dans le cas de la réinstallation, cela permet d'être présent là où les personnes sont en situation de danger sans avoir à effectuer des traversées extrêmement dangereuses. On peut aussi plus facilement identifier des besoins de protection. Je pense donc qu'elles font partie de l'avenir. Mais cela ne peut pas être exclusif d'un fonctionnement normal du droit d'asile. Or, j'ai quelques inquiétudes sur ce point. On constate en effet des tentations européennes, notamment de la Commission européenne, de biaiser sur ce point. Cela ne me paraît pas une bonne chose. La tradition de l'Europe et de l'Europe démocratique, et les principes fondamentaux du droit d'asile, veulent qu'une personne qui arrive sur le sol européen soit accueillie et protégée en Europe après instruction de sa demande.

31 La France n'a pas encore une grande culture de la réinstallation. Est-ce une procédure qui se développe réellement et effectivement en France? Si cette procédure bénéficie du soutien de l'UNHCR, n'est-elle pas également une menace de plus sur le droit international des réfugiés? 
32 Je pense que le changement en France est réel. Nous sommes aujourd'hui, en Europe, le premier pays pour la réinstallation et la relocalisation. Dans le monde, le troisième pays pour la réinstallation. Nous avons donc réussi un vrai saut qualitatif et quantitatif, même s'il faut rester modeste car les chiffres sont faibles en absolu. On assiste donc à un véritable changement.

33 Mais, le choix de développer les procédures de réinstallation doit s'exercer dans le respect des principes fondamentaux du droit d'asile. L'accès au territoire français reste une décision du gouvernement et non de l'OFPRA qui, elle, reste soumise au respect du droit d'asile. Il faut bien rappeler que les personnes protégées dans le cadre de la réinstallation sont des personnes très vulnérables que nous ne voyons pas sur le sol national: femmes seules, personnes âgées, familles avec des enfants malades, personnes dans des situations administratives inextricables comme les Kurdes apatrides de Syrie. Mais, il faut que la procédure d'asile de droit commun reste la voie principale : la réinstallation ne peut être qu'une voie complémentaire de protection.

34 Avant même que la décision de la CJUE ne soit rendue, et seulement quelques jours après la publication des conclusions du rapporteur public Paolo Mengozzi, quel est votre regard sur la possibilité ou l'obligation d'émettre des visas humanitaires à l'égard de personnes risquant d'être soumises à des traitements inhumains et dégradants ou à de la torture ${ }^{1}$ ?

Il ne faut pas être dans la confusion : il faut bien distinguer ce qui relève de l'asile et ce qui n'en relève pas. Il faut distinguer les procédures de l'asile des autres dispositifs qui relèvent, soit de la politique migratoire, soit d'une approche humanitaire. Les différents dispositifs doivent donc être clairement identifiés et cloisonnés afin que l'on sache clairement ce qui relève du droit d'asile ou non. Si la confusion devait se développer, c'est le droit d'asile qui en paierait le prix à la sortie. Ce qui se développe un jour pour de bonnes intentions peut s'avérer au final très préjudiciable à plus ou moins long terme.

Comme vous l'avez déjà publiquement affirmé, il n'y a pas de crise de l'asile en France, au sens où il n'y a pas d'afflux massif de demandeurs d'asile, notamment de Syriens ou d'Afghans. Qu'est-ce qui explique la spécificité française?

37 Avec près de 85000 demandeurs d'asile en 2016, on n'est pas en crise. Cela n'empêche pas les difficultés pour accueillir et héberger les demandeurs d'asile. Cela n'empêche pas non plus des difficultés certaines pour les institutions de l'asile comme l'OFPRA qui reste sous tension même si nous progressons en termes de délais. Il reste encore 20000 demandes qui attendent depuis plus de trois mois.

Mais, je constate que nous sommes déjà en train de revenir à une situation plus traditionnelle. Si on prend l'Allemagne, elle a accueilli à certaines périodes de son histoire, comme en 2015, massivement. Mais, sur la durée, l'Allemagne accueille moins que la France. On voit donc à présent les situations se rapprocher et se normaliser.

De mon point de vue, si les personnes sont davantage allées en Allemagne qu'en France, c'est pour deux raisons principales: la situation de l'emploi et la présence de compatriotes. Mais je pense que la durée des délais d'instruction en France a certainement joué un rôle également important. En effet, j'ai fait le constat très tôt que des délais d'instruction trop longs, comme c'était le cas en France jusqu'à il y a deux ans, découragent les personnes qui ont besoin de protection comme les Syriens. A l'inverse, je constate que cela encourage les personnes qui ont moins besoin de 
protection à venir. C'est la raison pour laquelle je suis très attaché à la réduction des délais d'instruction, tout en veillant à leur adaptation à la spécificité de chaque demande. Dans certains cas, comme les personnes victimes de tortures, les femmes victimes de traites, les homosexuels, il faut prendre du temps.

Je ne me prononcerai pas pour savoir si allonger les procédures d'asile était un choix politique délibéré. Mais, il existe une certaine forme de culture administrative et politique qui a conduit à l'allongement des délais. Or, cet allongement des délais nuit au droit d'asile et ne dissuade, au final, que ceux qui ont réellement besoin de protection. Cette culture administrative, sans avoir totalement disparue, est en recul massif depuis quatre ans et c'est dans ce sens que les réformes de l'OFPRA sont menées. C'est une erreur du point de vue de la dignité des gens, du point de vue du droit et du point de vue de l'efficacité d'avoir des délais d'instruction trop longs. Ce qui est efficace, c'est d'accueillir les personnes dans de bonnes conditions, d'instruire rapidement leurs demandes d'asile de manière à ce qu'elles puissent être rapidement fixées sur leur situation juridique et administrative. C'est dans cette voie qu'il faut continuer à progresser.

\section{NOTES}

1. CJUE, X \& C c . Belgique, C-638/16, 7 mars 2017. 